\title{
Actualización de la Modelación Numérica del Flujo de Escombros Producto de una Erupción del Volcán Cotopaxi - Flanco Norte
}

\author{
Toapaxi, Jorge ${ }^{1}$; Torres, Cristina ${ }^{1}$; Hamad, Khaled ${ }^{1}$; Vera, Pablo ${ }^{1}$; Méndez, Javier ${ }^{1}$

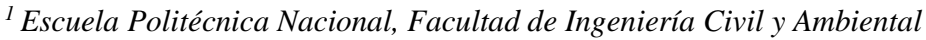

\begin{abstract}
Resumen: La actividad eruptiva del volcán Cotopaxi representa una amenaza latente para los miles de habitantes de ciudades aledañas a los drenajes naturales y la infraestructura relacionada con actividades productivas en el valle interandino de la zona central del Ecuador. La disponibilidad de información actualizada, relativa a modelos digitales de terreno, con resolución aceptable y sistemas computacionales que permiten obtener resultados prácticos en tiempos relativamente pequeños, hace posible la modelación numérica del flujo de escombros, considerando coeficientes de resistencia al movimiento que permiten la calibración del modelo en base a vestigios de eventos eruptivos históricos.
\end{abstract}

Palabras clave. Cotopaxi, Lahar primario, Modelo numérico, Sangolquí.

\section{Update of Numerical Simulation of Debris Flow Product of an Eruption of the Cotopaxi Volcano - Northern Sector}

\begin{abstract}
The eruptive activity of the Cotopaxi volcano represents a latent threat to thousands of inhabitants of cities bordering natural drainage and infrastructure related to productive activities in the central highlands of Ecuador. The availability of updated information on digital terrain models with acceptable resolution and computational systems that allow practical results to be obtained in relatively small times, makes possible the numerical modeling of lahar flow considering coefficients of motion resistance that allow the calibration of the model in base to vestiges of historical eruptive events.
\end{abstract}

Keywords. Cotopaxi, Numerical model, Primary lahar, Sangolquí.

\section{INTRODUCCIÓN}

El Cotopaxi es un volcán activo que tiene erupciones devastadoras, con periodo recurrente de alrededor de un siglo. La última erupción que ocurrió en el año de 1877, destruyó parcialmente Latacunga y llegó hasta el valle de los Chillos. Actualmente, las zonas amenazadas son altamente pobladas y de gran interés económico para el Ecuador, por esta razón, es necesario determinar con mayor precisión las zonas susceptibles al flujo de lahares (flujos de lodo, rocas, y escombros mezclados con agua provocados por la fusión casi instantánea de volúmenes de hielo importantes) (Cáceres, y otros, 2004).

En el año 2004, se realizó la modelación numérica unidimensional en el flanco norte del volcán, utilizando un modelo digital de terreno (MDT) con resolución espacial de 30 por 30 metros de tamaño de pixel (EMAAP \& EPN, 2004). En la presente investigación, se utiliza un MDT con resolución de 3 por 3 metros de tamaño de celda, lo cual, permitirá representar con mayor especificidad las zonas analizadas, aumentando la fiabilidad de resultados. De igual manera, se utiliza información del área del glaciar, procedente de estudios recientes (Cáceres B. , 2010) y se aplican herramientas computacionales de última generación, tales como: HEC-RAS 5.0, IBER 2.3 y FLOW 3D 11.1.
La modelación contempla una longitud de cauce de alrededor de 65 kilómetros, donde posiblemente el flujo de escombros, producto de una erupción, cruce las conducciones de tres sistemas de suministro de agua potable para la ciudad de Quito (Pita - Tambo, La Mica y Papallacta), lo cual pone en riesgo el abastecimiento del recurso hídrico para el $80 \%$ de la población. Además, existen varias zonas con alta densidad poblacional, importantes vías de comunicación, numerosas industrias y fábricas.

\section{OBJETIVOS}

- Actualizar la modelación numérica unidimensional del flujo generado por un evento eruptivo en el flanco norte del volcán Cotopaxi, hasta la zona densamente poblada de Sangolquí.

- Calibrar el modelo numérico unidimensional con base en los vestigios de la erupción del 26 de junio de 1877 .

- Determinar los niveles y zonas de inundación producidas por el flujo de escombros.

- Analizar bidimensionalmente el comportamiento del flujo en un tramo de la zona densamente poblada de Sangolquí. 


\section{METODOLOGÍA}

\subsection{Base Teórica}

El análisis de problemas de Mecánica de Fluidos se realiza mediante: la investigación experimental, el desarrollo teórico y la aplicación de la metodología de Dinámica de Fluidos Computacional (CFD: por sus siglas en inglés Computational Fluid Dinamic); dichos métodos, se relacionan y complementan entre sí. En lo referente a la metodología CFD, se fundamenta en lo siguiente (Carrillo, 2014):

- Ecuaciones generales de conservación a una partícula de fluido, cuyos principios y formulaciones son: principio de conservación de la masa, conservación de la cantidad de movimiento o segunda ley de Newton $\rightarrow$ Ecuaciones de Navier-Stokes (si se tienen en cuenta los términos viscosos y de disipación de energía) o ecuaciones de Euler (si se desprecian) y principio de conservación de la energía.

- Ecuación de estado o de comportamiento del fluido.

- Ecuaciones constitutivas del medio.

En lo que respecta al procedimiento para resolver un problema de mecánica de fluidos mediante la metodología CFD, se presentan los siguientes pasos (Blanco, 2007):

- Especificación de la geometría del problema.

- Creación del mallado o celdas en las que van a ser calculadas todas las variables.

- Definición de los modelos que se van a utilizar: modelos de turbulencia.

- Especificación de las propiedades del fluido: viscosidad, densidad, propiedades térmicas, etc.

- Imposición de las condiciones de contorno que controlan los valores de ciertas variables en los límites del dominio.

- Introducción de las condiciones iniciales.

- Control de los parámetros que afectan a la resolución numérica del problema.

- Proceso de cálculo.

- Análisis de la solución.

Por otro lado, es importante mencionar que los modelos bidimensionales (2D) estudian la variación de la velocidad promediada en vertical, considerando un campo de velocidades horizontal, es decir, cada uno de los puntos del dominio de estudio analiza el calado y las dos componentes de la velocidad.

Los modelos 2D resuelven las ecuaciones que se fundamentan en los principios básicos de la hidrodinámica, como son: la conservación de masa y la conservación de la cantidad de movimiento. Las ecuaciones implementadas en estos modelos se rigen a estos principios y toman el nombre de las ecuaciones de Saint Venant para aguas someras, las mismas que resultan de la deducción matemática de las ecuaciones tridimensionales de Reynolds; estas últimas, obtenidas a partir de las ecuaciones de Navier Stokes. Las hipótesis consideradas son las siguientes:
- La escala en profundidad es mucho menor a la escala horizontal.

- Distribución hidrostática de presiones.

Por lo tanto, un modelo numérico bidimensional es aplicable para el análisis de las variables hidráulicas de un cauce, cuando:

- El cauce presenta meandros con extensas zonas de inundación.

- El cauce presenta apreciables ensanchamientos y estrechamientos de sección que provoque zonas de recirculación y consecuente reducción de la sección hidráulica.

- Se evidencia un campo de velocidades heterogéneo por sección transversal.

\subsection{Modelación Unidimensional}

En la aplicación de la modelación unidimensional, para el cálculo de las variables hidráulicas (calado y velocidad), se utilizó el software libre HEC-RAS 5.0, desarrollado por el Hydrologic Engineering Center del US Army Corps of Engineers, el cual, nos permite simular flujos en cauces naturales o canales artificiales.

La revisión de la información cartográfica para el área de estudio, se compone de: redes hidrográficas a escala 1:50000, curvas de nivel cada 5 metros en formato .shp y .dwg, modelo digital del terreno de tamaño del pixel 3 por 3 metros en formato .tiff, curvas de nivel del cráter cada 5 y 10 metros (IGM) y orto-fotografías a escala 1:50000 (MAGAP-PRAT, SIGTIERRAS).

El drenaje norte se compone de los sistemas hidráulicos representados por los ríos Pita y El Salto, en las cercanías al volcán; y por los ríos Santa Clara y San Pedro, ubicados a media y larga distancia del volcán, respectivamente. Con este antecedente, se examina la información referente a los hidrogramas de los ríos Pita, El Salto y Santa Clara; así como también, en la zona de amenaza del lahar hacia el flanco norte, se revisa los datos de campo de los vestigios de la erupción de 1877 (EMAAP \& EPN, 2004).

\section{Escenarios eruptivos}

Considerando los dinamismos eruptivos, el estado y tamaño del glaciar, la magnitud de las erupciones precedentes y el comportamiento de otros volcanes análogos al Cotopaxi; se presenta cuatro posibles escenarios en el caso de una erupción del volcán Cotopaxi, ver Tabla 1. 
Tabla 1. Escenarios eruptivos.

\begin{tabular}{|c|c|c|c|c|}
\hline Escenario & VEI & $\begin{array}{c}\text { Tipo de } \\
\text { Actividad }\end{array}$ & Fenómenos Eruptivos & $\begin{array}{r}\text { Espesor } \\
\text { Promedio } \\
\text { Afectado } \\
\text { (metros) } \\
\end{array}$ \\
\hline $\begin{array}{c}1 \\
\text { (pequeño) }\end{array}$ & $1-2$ & $\begin{array}{l}\text { - Fuente de lava } \\
\text { - Estromboliana }\end{array}$ & $\begin{array}{l}\text { - Caídas de ceniza } \\
\text { - Fragmentos balísticos } \\
\text { - Flujos piroclásticos } \\
\text { pequeños }\end{array}$ & 0.3 \\
\hline $\begin{array}{c}2 \\
(\text { moderado) }\end{array}$ & $2-3$ & $\begin{array}{l}\text { - Estromboliana } \\
\text { - Vulcaniana }\end{array}$ & $\begin{array}{l}\text { - Caídas de ceniza } \\
\text { - Flujos piroclásticos } \\
\text { - Flujos de lava }\end{array}$ & $1-2$ \\
\hline $\begin{array}{c}3 \\
\text { (grande) }\end{array}$ & $3-4$ & $\begin{array}{l}\text { - Vulcaniana a } \\
\text { pliniana }\end{array}$ & $\begin{array}{l}\text { - Caídas de ceniza } \\
\text { - Flujos piroclásticos }\end{array}$ & 4 \\
\hline $\begin{array}{c}4 \\
\text { (muy } \\
\text { grande) }\end{array}$ & $\tilde{\mathrm{N}}>4$ & - Pliniana & $\begin{array}{l}\text { - Caídas de ceniza } \\
\text { - Flujos piroclásticos }\end{array}$ & 8 \\
\hline
\end{tabular}

(Fuente: EMAAP, y EPN. Evaluación de Impactos sobre la Infraestructura de la EMAAP-Q y de la Factibilidad de Obras de Mitigación. Quito, 2004)

En el estudio se consideró el Escenario 3, el mismo que se encuentra asociado a un Índice de Explosividad Volcánica 4 (VEI 4: por sus siglas en inglés). Este escenario se caracteriza por presentar una alta probabilidad de ocurrencia (56\%), donde el evento es explosivo con emisiones importantes de ceniza, flujos piroclásticos por el desbordamiento del magma desde el cráter y la formación de lahares con derretimiento del glaciar (EMAAP \& EPN, 2004).

La cuantificación del volumen de agua generado durante una erupción, se determina en base a: la proyección del tamaño del glaciar, área igual a $3.96 \mathrm{~km}^{2}$ (Cáceres B. , 2010) y la disminución promedio del espesor del glaciar, obtenido de los estudios realizados en otros volcanes, como: el nevado del Ruiz de Colombia, con 3 metros de espesor (Thouret, 1990) y el Monte Santa Elena de Estados Unidos, con 6 metros (Brugman \& Meier, 1981).

El tamaño del glaciar del Cotopaxi ha sido estudiado partiendo del análisis de las cuencas glaciares, estudiadas a partir de fotografías aéreas y controles de campo. Para lo cual, se dividió el glaciar en diecinueve secciones, que suman $21.8 \mathrm{~km}^{2}$ (año 1976). El drenaje norte se compone de las primeras seis cuencas: Sindipamba, Carnero Machay, Potrerillos, Pucahuaycu Norte, Mudadero y Cajas (Jordan, 1976).

Estudios recientes sobre el estado del casquete glaciar, presentan una disminución importante del área debido a múltiples factores como el calentamiento global (Cáceres B. , 2010), por esta razón, en un evento eruptivo se produciría una significativa reducción del caudal del flujo de escombros.

Los hidrogramas de entrada son de forma triangular con el máximo valor en el primer tercio de la duración total del evento, en el orden de los 30 a 40 minutos (EMAAP \& EPN, 2004). Para el ingreso de estos datos, se consideró las secciones de entrada "Hostería Tambopaxi" para el río Salto y "Hacienda San Rafael" para el río Pita; en la siguiente figura se muestran los hidrogramas de ingreso.

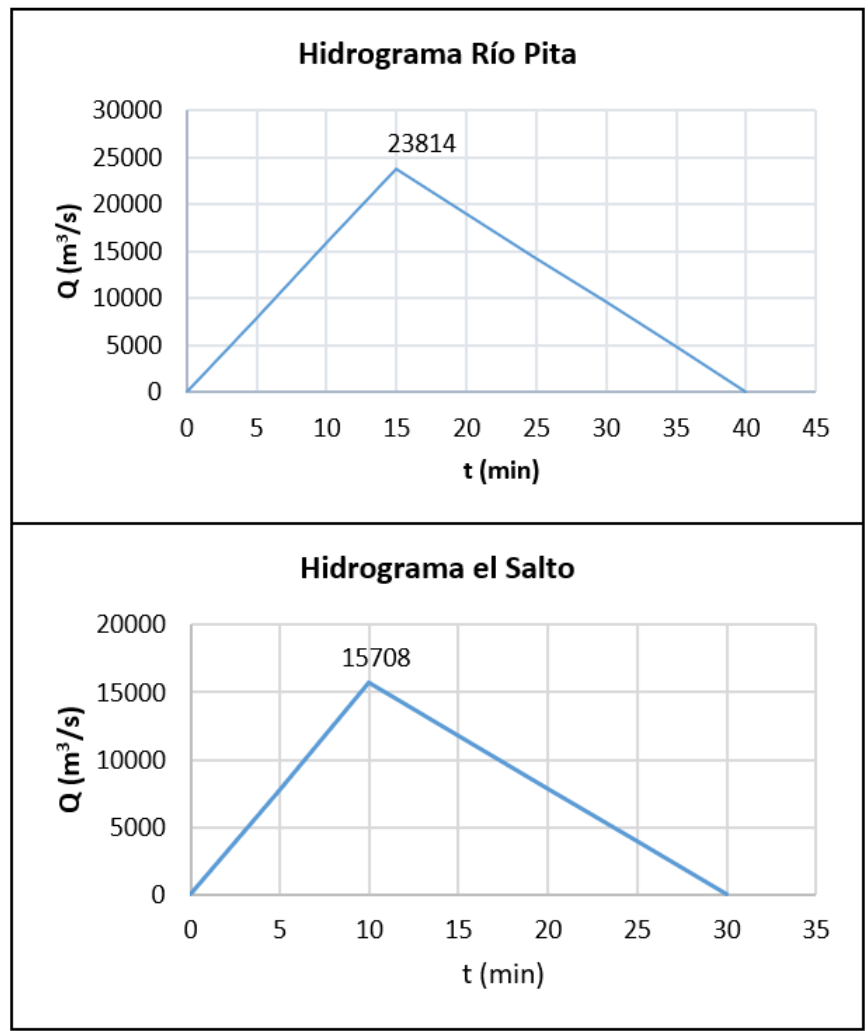

Figura 1. Hidrograma de entrada para el Escenario 3 - 2011.

Entre febrero y marzo del año 2004, el personal del Instituto Geofísico de la Escuela Politécnica Nacional realizó el levantamiento de información de los vestigios de los lahares del evento eruptivo de junio de 1877, para el Estudio de amenaza volcánica del proyecto: "EVALUACIÓN DE IMPACTOS SOBRE LA INFRAESTRUCTURA DE LA EMAAP-Q Y ESTUDIO DE FACTIBILIDAD DE LAS OBRAS DE MITIGACIÓN ANTE UNA ERUPCIÓN DEL VOLCÁN COTOPAXI". En este trabajo de campo se levantó información de calado, perímetro mojado, velocidad media y caudal pico, en: 26 secciones del río Pita, 10 secciones del río Salto, 8 secciones del río Santa Clara y 13 secciones del río San Pedro.

En lo referente a la calibración, ésta se lleva a cabo con el modelo unidimensional en flujo permanente, en base a los datos de campo recopilados a partir de los vestigios del evento eruptivo de 1877 (Escenario 3). Los parámetros de calibración corresponden a los calados o profundidades de flujo, asociados a los caudales máximos de crecida en cada tramo de análisis.

El tramo de simulación unidimensional es de aproximadamente $50 \mathrm{~km}$ de longitud, desde la sección "Hostería Tambopaxi" hasta la sección "La Armenia" en el río San Pedro, este último se encuentra aguas abajo de la junta de los ríos Santa Clara y Pita en el sector de "El Triángulo" en Sangolquí, ver Figura 2. 


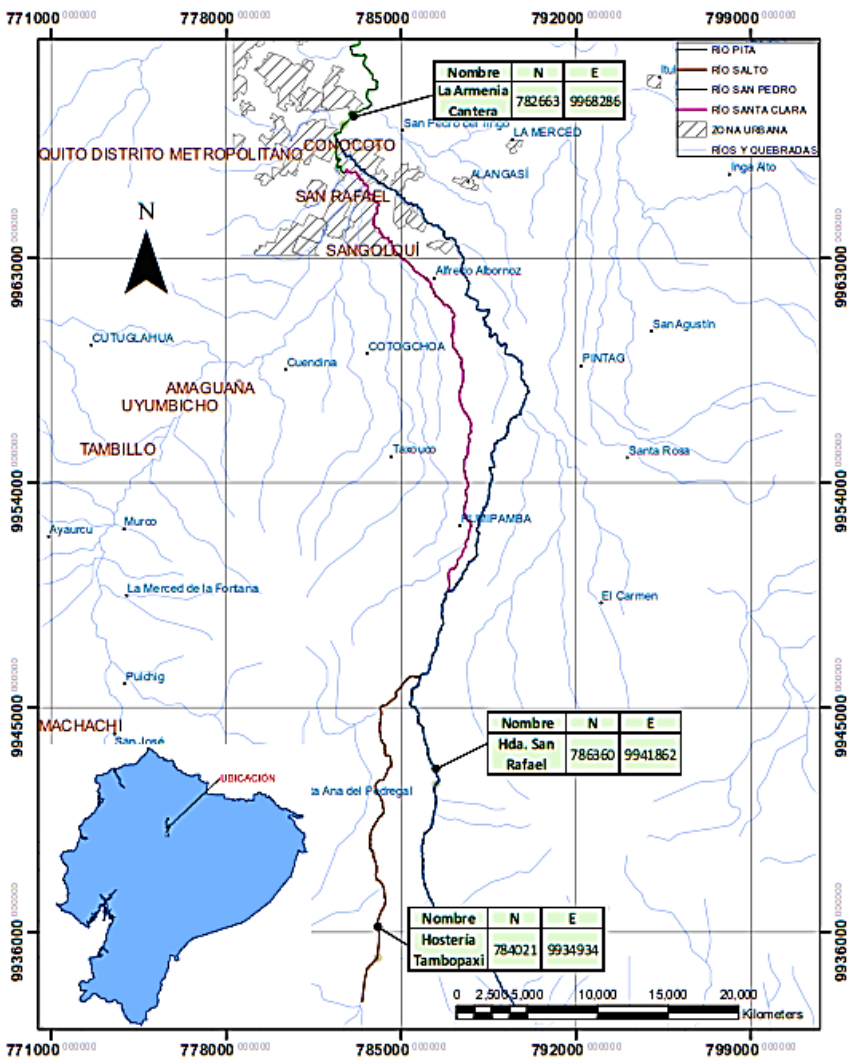

Figura 2. Tramo para la actualización de la modelación numérica unidimensional.

\subsection{Modelación bidimensional}

La modelación numérica bidimensional se realizó en la zona densamente poblada de Sangolquí. Para esta modelación se utilizó los programas computacionales IBER 2.3 y FLOW 3D 11.1. IBER es un software libre bidimensional desarrollado por el Grupo de Ingeniería del Agua y del Medio Ambiente, GEAMA (Universidad de A Coruña, UDC) y el Instituto FLUMEN (Universitat Politècnica de Catalunya, UPC, y Centro Internacional de Métodos Numéricos en Ingeniería, CIMNE), y se aplica a modelos numéricos en hidráulica y morfología fluvial. Por otro lado, FLOW-3D es un software de simulación de fluidos computacional CFD, creado por FlowScience y aplicado principalmente al cálculo de fluidos a superficie libre, el mismo que dispone de la opción Shallow Water (aguas poco profundas), que de igual manera, realiza el cálculo como un modelo bidimensional.

El tramo de esta modelación comprende $36.36 \mathrm{~km}^{2}$, dentro de los cuales se encuentran los ríos: Pita 7.83 km, Santa Clara 5.1 km y San Pedro 3.8 km.

En las modelaciones se aplica el siguiente procedimiento:

- Para el ingreso de la topografía a los CFDs, se extrae el tramo a simular del modelo digital del terreno (mdt), aplicando un software de sistemas de información geográfica (SIG) y se transforma esta información a formato ASCII.

- $\quad$ Se genera un mallado estructurado hexaédrico de 9 por 9 metros, donde cada malla presenta al final un único hidrograma de salida. Se crea tres bloques de malla con planos de ingreso de caudal en los ríos Pita y Santa Clara, y con salida de flujo en el río San Pedro de acuerdo a los drenajes presentes en el tramo.

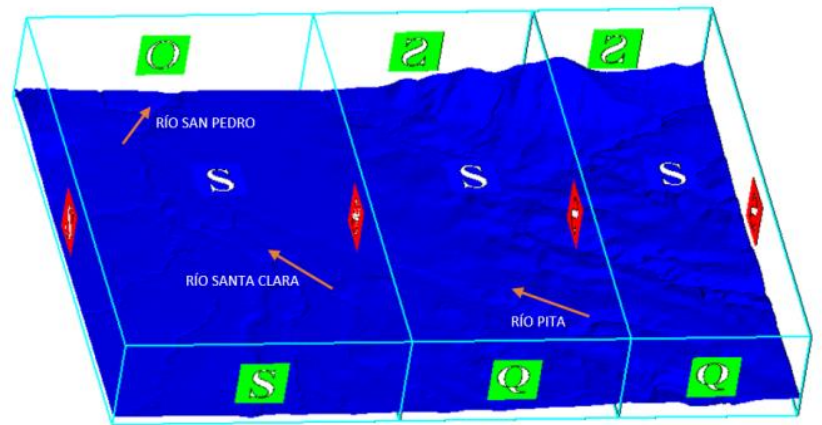

Figura 3. Bloques de malla en el tramo de análisis

- La reología del flujo de escombros se representa a través de: el coeficiente de rugosidad de Manning (n) en el programa IBER y con la rugosidad absoluta en el programa FLOW 3D. Estos coeficientes de resistencia al movimiento además de ser un parámetro que representa la superficie del cauce, representa el comportamiento de la mezcla agua-escombros.

- $\quad$ En el modelo IBER se utilizó un coeficiente de rugosidad de Manning (n) de 0.063, resultado de la ponderación de los coeficientes de resistencia al movimiento, según la longitud del río (EMAAP \& EPN, 2004). En el programa FLOW 3D, se utiliza un valor de rugosidad absoluta de 0.216 metros, obtenido del estudio: "PREPARACIÓN DE LA BASE GEOMORFOLÓGICA PARA LA SIMULACIÓN NUMÉRICA TRIDIMENSIONAL DEL FLUJO DE LAHARES DEL VOLCÁN COTOPAXI, FLANCO NORTE", realizado en el año 2016 en la Escuela Politécnica Nacional. (BID \& EPN, 2016)

- El ingreso de fluido se configura como agua, con el vector de dirección en cada río. Y los hidrogramas de ingreso se obtuvieron de la modelación unidimensional.

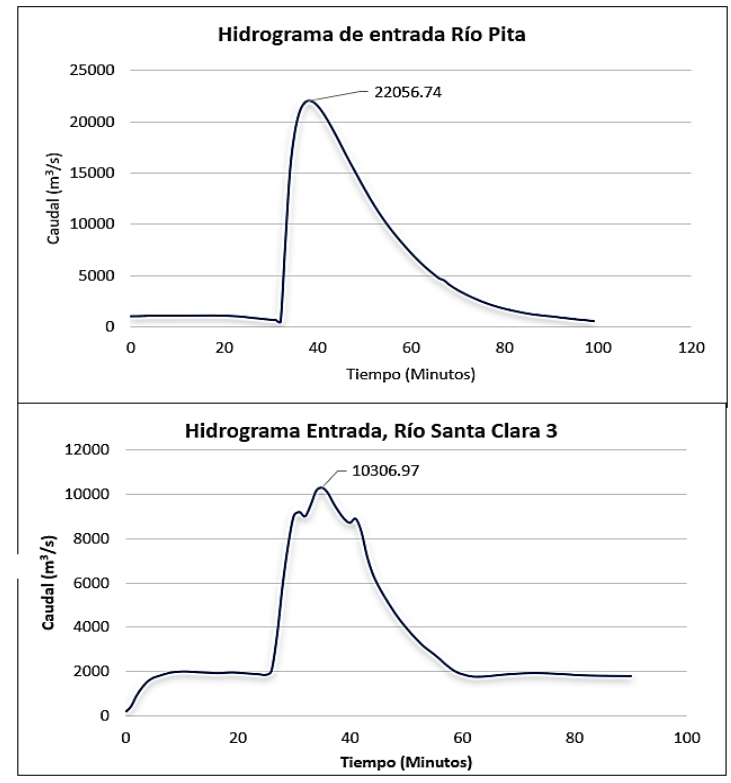

Figura 4. Hidrogramas de ingreso.

En el análisis bidimensional se consideró cinco secciones, cuyas coordenadas se indican en la siguiente tabla: 
Tabla 2. Secciones de Análisis de resultados.

\begin{tabular}{|c|c|c|c|c|c|}
\hline \multirow{3}{*}{ Sección } & \multirow{3}{*}{ Río } & \multicolumn{4}{|c|}{ Coordenadas UTM } \\
\hline & & \multicolumn{2}{|c|}{ Margen Izquierda } & \multicolumn{2}{|c|}{ Margen Derecha } \\
\hline & & $\mathbf{X}$ & $\mathbf{Y}$ & $\mathbf{X}$ & $\mathbf{Y}$ \\
\hline \multirow[b]{2}{*}{1} & Santa & 783262.9 & 9963579.2 & 784742.2 & 9964419.8 \\
\hline & Clara & 1 & 1 & 8 & 9 \\
\hline \multirow[t]{2}{*}{2} & $\begin{array}{c}\text { Santa } \\
\text { Clara- } \\
\text { Pita }\end{array}$ & $\begin{array}{c}782659.6 \\
3\end{array}$ & $\begin{array}{c}9965556.7 \\
8\end{array}$ & $\begin{array}{c}785281.6 \\
7\end{array}$ & $\begin{array}{c}9966198.9 \\
1\end{array}$ \\
\hline & San & 781566.5 & 9968302.7 & 784785.6 & 9967753.6 \\
\hline \multirow[t]{2}{*}{3} & Pedro & 1 & 9 & 3 & 8 \\
\hline & & 786203.5 & 9963617.8 & 786479.0 & 9965408.7 \\
\hline \multirow[t]{2}{*}{4} & Pita & 1 & 1 & 5 & 6 \\
\hline & & 784765.4 & 9964692.8 & 785493.6 & 9966017.6 \\
\hline 5 & Pita & 8 & 9 & 3 & 1 \\
\hline
\end{tabular}

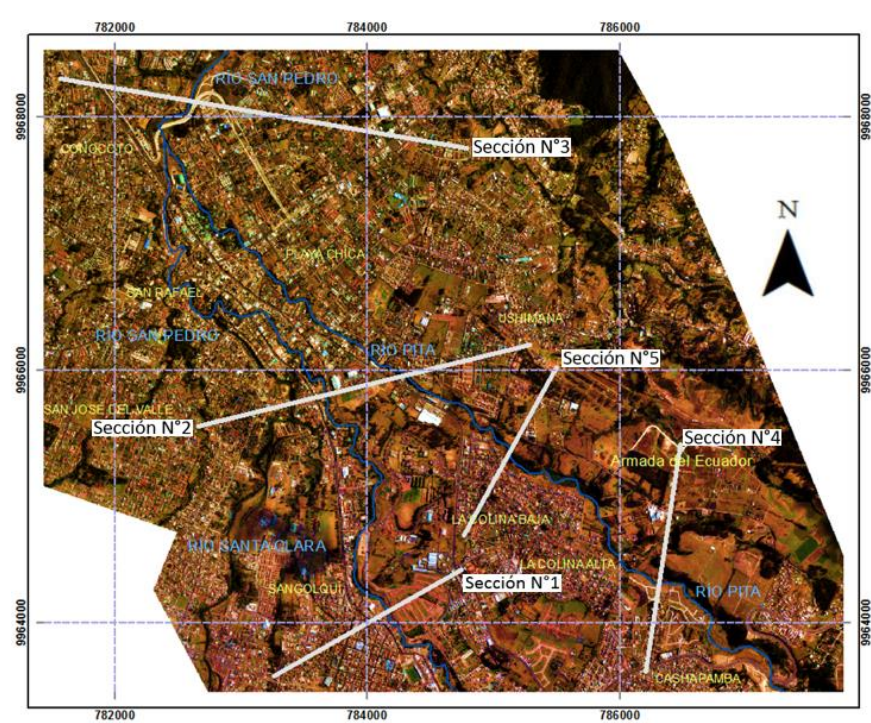

Figura 5. Ubicación de las secciones de análisis.

\section{RESULTADOS}

\subsection{Modelación Unidimensional}

La calibración del modelo unidimensional se alcanza representando los niveles de vestigios del evento de 1877 , cuyos coeficientes de resistencia al movimiento (n) varían entre 0.01 y 0.09

El análisis del flujo no permanente permite determinar los caudales de crecida en las secciones de entrada y salida del modelo numérico, los mismos que, debido a la laminación del flujo, van disminuyendo conforme transita el caudal a través del cauce.

De igual manera, en la siguiente tabla se presenta el tiempo en el cual se registra el caudal máximo y la duración del hidrograma. Además, se calculan los calados con el modelo unidimensional obteniéndose valores que fluctúan entre 23 y 49 metros de altura.
Tabla 3.Tramo para la actualización de la modelación numérica

\begin{tabular}{ccccc}
\multicolumn{5}{c}{ unidimensional. } \\
\hline Tramo & Tipo & $\begin{array}{c}\text { Caudal } \\
\text { máximo } \\
\mathbf{m}^{\mathbf{3}} / \mathbf{s}\end{array}$ & $\begin{array}{c}\text { Tiempo } \\
\text { (minutos) }\end{array}$ & $\begin{array}{c}\text { Duración } \\
\text { (minutos) }\end{array}$ \\
\hline Pita 1 & Entrada & 23814.0 & 13 & 40 \\
Pita 1 & Salida & 22769.7 & 17 & 60 \\
Salto 1 & Entrada & 15708.5 & 10 & 30 \\
Salto 1 & Salida & 14228.0 & 20 & 60 \\
Pita 2 & Entrada & 36997.7 & 20 & 60 \\
Pita 2 & Salida & 36692.3 & 21 & 60 \\
Santa Clara 1 & Entrada & 11007.7 & 21 & 60 \\
Santa Clara 1 & Salida & 9771.1 & 40 & 80 \\
Sangolquí-Ejido & Entrada & 10306.9 & 35 & 60 \\
Pita 3 & Entrada & 25684.6 & 21 & 60 \\
Pita 3 & Salida & 22056.7 & 38 & 80 \\
Colina & Entrada & 22056.7 & 38 & 80 \\
\hline
\end{tabular}

\subsection{Modelación Bidimensional}

\section{Alturas Máximas}

Tomando en consideración las modelaciones en IBER y FLOW 3D, en la siguiente tabla se compara para las secciones de análisis, los resultados de calados máximos; donde se obtiene un porcentaje de coincidencia de $90 \%$ en la margen izquierda y $85 \%$ en la margen derecha del río.

Tabla 4. Alturas máximas.

\begin{tabular}{|c|c|c|c|}
\hline \multirow[b]{2}{*}{ Sección } & \multirow[b]{2}{*}{ Río } & \multicolumn{2}{|c|}{ Calado (IBER) } \\
\hline & & Margen Izquierda & $\begin{array}{l}\text { Margen } \\
\text { Derecha }\end{array}$ \\
\hline 1 & Santa Clara & 11 & 16 \\
\hline 2 & Santa Clara-Pita & 5 & 15 \\
\hline 3 & San Pedro & 19 & 17 \\
\hline 4 & Pita & 18 & 12 \\
\hline \multirow[t]{2}{*}{5} & Pita & 16 & 15 \\
\hline & & \multicolumn{2}{|c|}{ Calado (FLOW-3D) } \\
\hline Sección & Río & Margen Izquierda & $\begin{array}{l}\text { Margen } \\
\text { Derecha }\end{array}$ \\
\hline 1 & Santa Clara & 11 & 15 \\
\hline 2 & Santa Clara-Pita & 4 & 12 \\
\hline 3 & San Pedro & 19 & 18 \\
\hline 4 & Pita & 15 & 7 \\
\hline \multirow[t]{2}{*}{5} & Pita & 18 & 15 \\
\hline & & \multicolumn{2}{|c|}{ Porcentaje de Coincidencia } \\
\hline Sección & Río & Margen Izquierda & $\begin{array}{l}\text { Margen } \\
\text { Derecha }\end{array}$ \\
\hline 1 & Santa Clara & 100 & 94 \\
\hline 2 & Santa Clara-Pita & 80 & 80 \\
\hline 3 & San Pedro & 100 & 94 \\
\hline 4 & Pita & 83 & 58 \\
\hline 5 & Pita & 89 & 100 \\
\hline & Promedio & 90 & 85 \\
\hline
\end{tabular}

\section{Velocidades Máximas}

Considerando las cinco secciones de análisis, en la siguiente tabla se observa que los resultados de las velocidades máximas obtenidas en las modelaciones con los dos programas, son muy similares. 
Tabla 5. Velocidades Máximas.

\begin{tabular}{lcc}
\hline & \multicolumn{3}{c}{$\begin{array}{c}\text { Velocidades Máximas (m/s) } \\
\text { IBER }\end{array}$} & FLOW 3D \\
\hline Sección 1 & 13 & 13 \\
Sección 2 & 14 & 13 \\
Sección 3 & 13 & 13 \\
Sección 4 & 18 & 19 \\
Sección 5 & 21 & 21 \\
\hline
\end{tabular}

\section{Hidrograma de Salida}

En lo referente a los hidrogramas de salida, en la siguiente figura se muestran los resultados obtenidos donde se identifica el caudal pico:
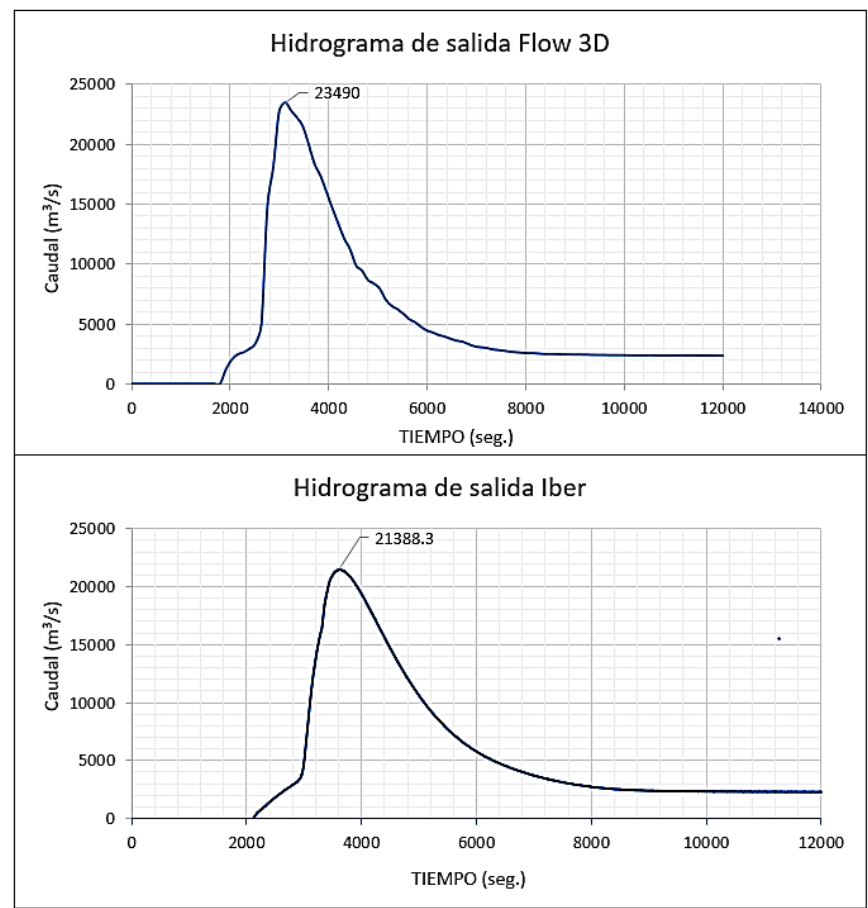

Figura 6. Hidrograma de salida, río San Pedro.

Por otro lado, se verifica los volúmenes de ingreso y salida de las modelaciones. En la siguiente tabla se muestran los valores obtenidos:

Tabla 6. Volumen de ingreso y salida al tramo de análisis.

\begin{tabular}{lccc}
\hline \multicolumn{1}{c}{ Río } & $\begin{array}{c}\text { Volumen de } \\
\text { Ingreso } \\
\left(\mathbf{M m}^{\mathbf{3}}\right)\end{array}$ & $\begin{array}{c}\text { Volumen de } \\
\text { Salida FLOW } \\
\text { 3D } \\
\left(\mathbf{M m}^{\mathbf{3}}\right)\end{array}$ & $\begin{array}{c}\text { Volumen de } \\
\text { Salida IBER } \\
\left(\mathbf{M m}^{\mathbf{3}}\right)\end{array}$ \\
\hline Santa & 17.6 & - & - \\
Clara & 29.97 & - & - \\
Pita & - & 44.31 & 43.17 \\
San Pedro & $\mathbf{4 7 . 5 7}$ & $\mathbf{4 4 . 3 1}$ & $\mathbf{4 3 . 1 7}$ \\
TOTAL & & & \\
\hline
\end{tabular}

\section{Área de inundación}

En la siguiente figura anterior se observa los mapas de inundación en la zona densamente poblada de Sangolquí. El área de inundación en el tramo de análisis, resultado de la modelación en IBER es de $8.26 \mathrm{~km}^{2}$, mientras que en la modelación en FLOW 3D es de 7.72 km²

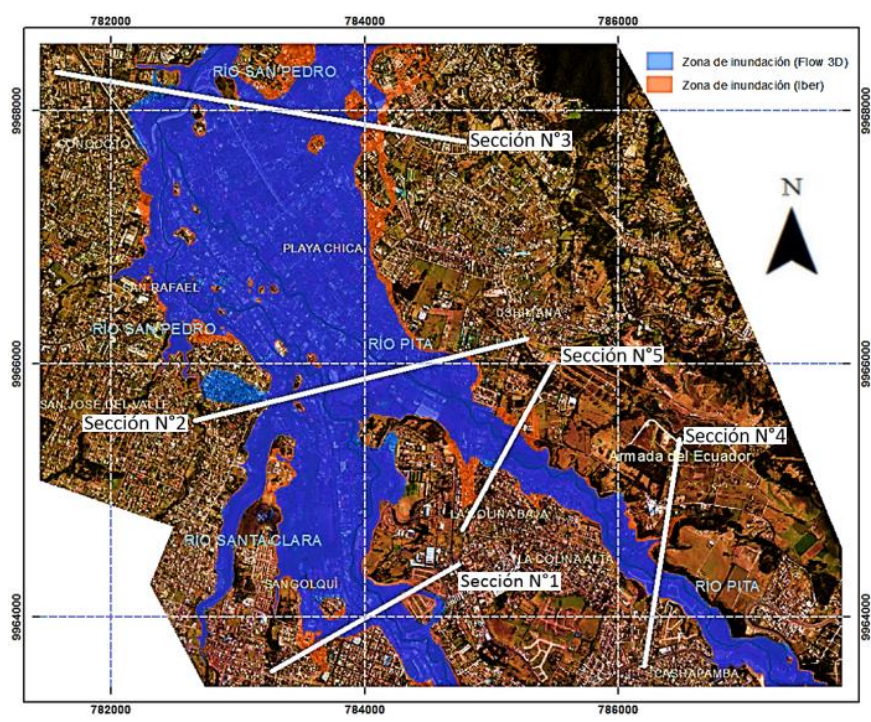

Figura 7. Zona de Inundación.

\section{CONCLUSIONES}

El coeficiente de rugosidad " $n$ " de Manning, que en este caso, representa las condiciones de resistencia al movimiento, tanto del lahar con el contorno, como las internas de la mezcla; es el parámetro que permitió realizar la calibración del modelo unidimensional, al comparar los calados obtenidos en la modelación, con los vestigios del evento eruptivo de 1877.

Se determinó que al utilizar un mallado estructurado hexaédrico de 9 por 9 metros, se obtienen resultados aceptables, en comparación al mallado más fino de 3 por 3 metros; lo cual permite: simplificar el modelo, reducir los recursos computacionales y los tiempos de cálculo.

Para el evento de mayor probabilidad de ocurrencia, escenario 3, al considerar el área del casquete glaciar al año 2010, se verificó que la afectación en el caso que se produzca un evento eruptivo, es menor en comparación a los resultados obtenidos en estudios anteriores.

Con base en la comparación de parámetros como: el calado, la velocidad y el área de afectación; se concluye que los resultados obtenidos al aplicar los modelos IBER y FLOW 3D, son muy similares. En general, considerando el calado de los dos modelos, se identificó una coincidencia de: $90 \%$ en la margen izquierda y $85 \%$ en la margen derecha del cauce.

Respecto a los resultados de velocidades máximas, existe un porcentaje de coincidencia de $98 \%$, cuyo valor de velocidad máxima para la sección representativa es de $21 \mathrm{~m} / \mathrm{s}$ en las dos modelaciones.

El caudal pico del hidrograma de salida es de $21.388 \mathrm{~m} / \mathrm{s}$ a los 60 minutos y 23.490 a los 52 minutos, para las modelaciones con IBER Y FLOW 3D, respectivamente; alcanzando un $92 \%$ de similitud. El volumen de salida es de 43.17 millones de metros cúbicos en el modelo IBER y 44.31 millones de metros cúbicos en el modelo FLOW 3D, coincidiendo en un $97 \%$.

En lo referente a la comparación de las áreas de afectación, estas son similares y aceptables, el área de inundación que 
resulta de la modelación numérica en IBER es de $8.26 \mathrm{~km}^{2}$ y en el FLOW 3D es de $7.72 \mathrm{~km}^{2}$, presentando un $93.5 \%$ de coincidencia.

Se recomienda el uso del modelo unidimensional en zonas con altas pendientes de fondo o encañonadas (encauzadas), es decir, donde predomine la dirección longitudinal del flujo del río, parar lograr la representación del modelo numérico de manera satisfactoria y optimizar recursos.

En cambio, en zonas susceptibles a inundación, como es el caso de la zona densamente poblada de Sangolquí, se recomienda el uso de un modelo bidimensional, que permita representar el flujo en dos direcciones y consiga una caracterización adecuada del fenómeno.

Con la finalidad de obtener resultados fiables en tiempos razonables, al realizar la modelación numérica bidimensional y tridimensional, se sugiere emplear computadoras de gran capacidad de procesamiento y almacenamiento. Se recomienda las siguientes características: Core I7 3.4 GHZ, 8 núcleos, 32 GB RAM, espacio disponible en disco 200 GB $\mathrm{SSD}$, tarjeta gráfica de video $2 \mathrm{~GB}$; o superior.

\section{REFERENCIAS}

Academic Program-FLOW Science. (2015). Hybrid Shallow Water/3D Flow. Obtenido de

https://www.flow3d.com/modeling-capabilities/hybridshallow-water-3d-flow/

BID, \& EPN. (2016). Preparación de la Base Geomorfológica para la Simulación Tridimensional del Flujo de Lahares en el Flanco Norte del Volcán Cotopaxi. Quito.

Blanco, E. (2007). Simulación numérica de flujos (CFD) con el programa FLUENT.

Brugman, M., \& Meier, M. (1981). Response of glaciers to the eruption of Mount St. Helens. En Lipman P.W. \& Mullineaux D.R. (eds.). The 1980 eruptions of Mount St. Helens, Washington. US. Geol. Surv. Prof. Paper 1250, 743-756.

Cáceres, B. (2010). Actualización del inventario de tres casquetes glaciares del Ecuador.

Cáceres, B., Ramírez, J., Francou, B., Eissen, J., Taupin, T., Jordan, E., . . Mothes, P. (2004). Determinación del volumen del Casquete de hielo del Volcán Cotopaxi.

Carrillo, S. J. (2014). Metodología numérica y experimental para el diseño de los cuencos de disipación en el sobrevertido de presas de fábrica.

EMAAP, \& EPN. (2004). Evaluación de Impactos sobre la Infraestructura de la EMAAP-Q y de la Factibilidad de Obras de Mitigación. Quito.

FLOW Science. . (2015). FLOW-3D V11.1 User Manual.

Instituto Geofísico - EPN. (2015). Volcán Cotopaxi. Obtenido de http://www.igepn.edu.ec/

Jordan. (1976). Inventario de glaciares del volcán Cotopaxi.

Thouret, J.-C. (1990). Effects of the november 13, 1985 eruption on the snow pack ans ice cap of Nevado del Ruiz volcano, Colombia. Journal of Volcanology and Geothermal Research.

\section{BIOGRAFÍAS}

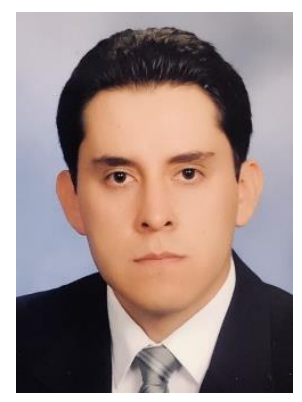

Jorge Augusto Toapaxi Álvarez, Ingeniero Civil, Magister en Recursos Hídricos con mención en "Diseño de Proyectos Hidráulicos" de la Escuela Politécnica Nacional (EPN). Profesor e Investigador de la Facultad de Ingeniería Civil y Ambiental - EPN desde 2015. Jefe del Laboratorio Docente de Hidráulica desde el 2016. Coordinador de la Maestría en Hidráulica de la EPN desde el 2018. Director de Proyectos de Investigación en las siguientes líneas: Mecánica Teórica y Computacional de Fluidos, Modelación Física de Fenómenos Hidráulicos, y Diseño de Obras Hidráulicas. Consultor de Proyectos Hidráulicos e Hidrológicos. Miembro de la Red de jóvenes investigadores de la IAHR.

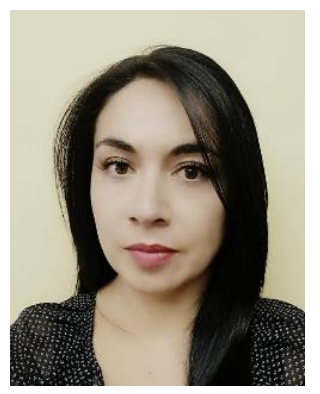

Cristina Alexandra Torres Jacobowitz, Ingeniera Civil, Magister en Recursos Hídricos con mención en "Diseño de Proyectos Hidráulicos" de la Escuela Politécnica Nacional. Experiencia en diseño y evaluación de obras hidráulicas, modelación física y numérica de fenómenos hidráulicos, diseño vial, programación y dirección de obras civiles. Actualmente trabaja como Profesora Ocasional a tiempo completo de la Facultad de Ingeniería Civil y Ambiental de la Escuela Politécnica Nacional. Sus intereses de investigación se enfocan en: Mecánica Teórica y Computacional de Fluidos, Modelación Física de Fenómenos Hidráulicos, Diseño de Obras Hidráulicas y Transporte de Sedimentos.

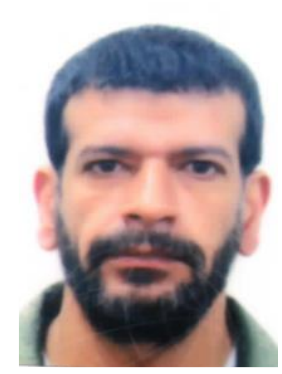

Khaled M. Hamad, Profesor de Ingeniería Hidráulica - Escuela Politécnica Nacional desde 2015, Ingeniero Civil - Universidad del Cairo y Escuela Politécnica Nacional 2008, Master en Ingeniería Hidráulica (MSc) - Escuela Politécnica Nacional 2011, y Doctor en Filosofía (Ph. D.) en Transporte de Sedimentos y Morfodinámica Fluvial - Escuela Superior Técnica de Puertos, Caminos y Canales de Barcelona - Universitat Politècnica de Catalunya - España 2015. Ha realizado investigaciones en transporte de sedimentos con el Dr. Jacob Odgaard - Universidad de Iowa USA, galardonado el mejor docente de la Facultad de Ingeniería Civil y Ambiental - EPN 2017 por el Colegio de Ingenieros Civiles de Pichincha. 


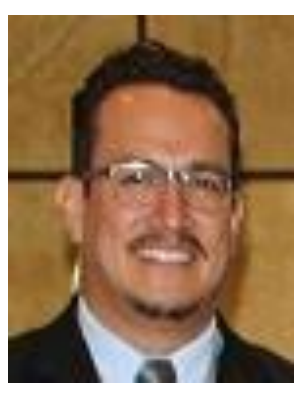

Pablo Alberto Vera Romero (1981).

Ingeniero Civil y Magister en Recursos Hídricos mención en Diseño de proyectos hidráulicos de la Escuela Politécnica Nacional. Profesor Ocasional a tiempo completo de la Facultad de Ingeniería Civil y Ambiental de la EPN en el área de Hidráulica. Actualmente es integrante del equipo técnico del Centro de Investigaciones y Estudios en Recursos Hídricos CIERHIEPN, desarrollando proyectos de investigación básica y aplicada. Miembro la Red de jóvenes investigadores y Editor asociado en la revista Hidrolatinoamericana de jóvenes investigadores y profesionales IAHR.

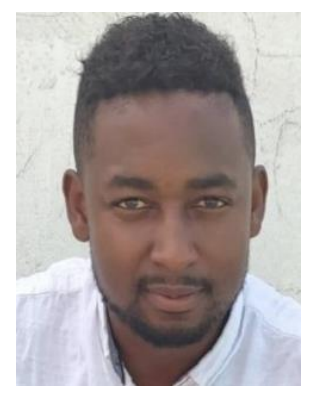

Javier Alexander Méndez Padilla, Nacido en Quito-Ecuador el 30 de junio de 1992, Sus estudios secundarios los cursó en el Colegio Nacional Eloy Alfaro de la misma ciudad, posteriormente consiguió el título de Ingeniero Civil con mención en Hidráulica en la Escuela Politécnica Nacional (2017).Formó parte del equipo de trabajo del Proyecto "Simulación Numérica del Flujo de Lahares Primarios hasta los tramos de implantación de las centrales hidroeléctricas San Francisco y Manduriacu”. Actualmente se desempeña como consultor de obras hidrosanitarias e hidráulicas y muestra un especial interés por la gestión de Recursos Hídricos. 